\title{
Localized modes on an Ablowitz-Ladik nonlinear impurity
}

\author{
Mario I. Molina \\ Departmento de Física, Facultad de Ciencias, \\ Universidad de Chile, Santiago, Chile
}

\begin{abstract}
We study localized modes on a single Ablowitz-Ladik impurity embedded in the bulk or at the surface of a one-dimensional linear lattice. Exact expressions are obtained for the bound state profile and energy. Dynamical excitation of the localized mode reveals exponentially-high amplitude oscillations of the spatial profile at the impurity location. The presence of a surface increases the minimum nonlinearity to effect a dynamical selftrapping.
\end{abstract}


The study of nonlinear dynamics in discrete systems has attracted a special attention recently due to novel physics and possible interesting applications [1]. Among these systems, we find the integrable discretized version of the continuum NLS equation, the so-called Ablowitz-Ladik (AL) equation[2]:

$$
i \frac{d C_{n}}{d t}+\left(V+\mu\left|C_{n}\right|^{2}\right)\left(C_{n+1}+C_{n-1}\right)=0
$$

This integrable version support moving, nonlinear, spatially-localized excitations in the form of lattice solitons, found through the use of the inverse scattering transform method. The AL equations constitute a starting point for many studies on the interplay of disorder, nonlinearity and discreteness. For instance, when examining the effects of disorder, a wellknown approach is to assume a perturbative approach and try to compute the evolution of the soliton parameters[3]. When the scale of the disorder is high, this approach is no longer tenable and one must resort to numerical schemes. On the other hand, when nonlinearity is large, the spatial soliton profile is well localized in space, meaning that only a small number of sites around the soliton center are effectively nonlinear. The system then looks very similar to a linear system containing a small cluster of nonlinear sites, or even a single nonlinear impurity. This simplified system is now amenable to exact mathematical treatment, and the influence of other potentially competing effects, such as dimensionality, boundary effects, noise, etc., can be more easily studied without losing the essential physics. This approach has been successfully used for the DNLS equation[4],

$$
i \frac{d C_{n}}{d t}+V\left(C_{n+1}+C_{n-1}\right)+\gamma\left|C_{n}\right|^{2} C_{n}=0,
$$

where it was predicted that, for a semi-infinite nonlinear chain, the presence of a surface would increase the amount of nonlinearity required to form a localized surface mode. This was subsequently observed in later studies [5, 6]. When used for the two-dimensional semiinfinite square lattice, this procedure predicted that this time, the presence of a boundary would decrease the minimum nonlinearity needed to create a surface localized mode[7]. This was later found to be the case[8].

In this Letter, we introduce a novel type of nonlinear defect in a one-dimensional discrete chain, this time using the framework of the AL equation (1).

We consider a one-dimensional array of linear sites, containing a single, Ablowitz-Ladik impurity located at site $n_{0}$. In the tight-binding framework, the evolution equation for the 
amplitude is given by

$$
i \frac{d C_{n}}{d t}+\left(V+\delta_{n, n_{0}} \mu\left|C_{n}\right|^{2}\right)\left(C_{n+1}+C_{n-1}\right)=0
$$

where $C_{n}$ is the complex amplitude at site $n, V$ is the nearest-neighbor coupling coefficient, and $\mu$ is the Ablowitz-Ladik (AL) parameter. We will be interested in stationary-state solutions of the form $C_{n}(t)=C_{n} \exp (i \omega t)$. This leads to the system of equations:

$$
-\omega C_{n}+\left(V+\delta_{n, n_{0}} \mu\left|C_{n}\right|^{2}\right)\left(C_{n+1}+C_{n-1}\right)=0
$$

From Eq.(3) it can be easily proven that the norm

$$
\mathcal{N}=(V / \mu) \log \left(1+(\mu / V)\left|C_{0}\right|^{2}\right)+\sum_{n}^{\prime}\left|C_{n}\right|^{2}
$$

is a conserved quantity, where the prime in the sum indicates that the sum is carried out over all sites, excepting the impurity site, $n=n_{0}$. We normalize the time to $\tau=V t$ and the probability amplitude to $\phi_{n}=C_{n} / \sqrt{\mathcal{N}}$. With these definitions, Eq.(3) simplifies to

$$
i \frac{d \phi_{n}}{d \tau}+\left(1+\delta_{n, n_{0}} \nu\left|\phi_{n}\right|^{2}\right)\left(\phi_{n+1}+\phi_{n-1}\right)=0
$$

where $\nu \equiv \mathcal{N} \mu / V$. The normalization condition becomes

$$
1=(1 / \nu) \log \left(1+\nu\left|\phi_{n_{0}}\right|^{2}\right)+\sum_{n}^{\prime}\left|\phi_{n}\right|^{2}
$$

The equation for the stationary state, acquires now its dimensionless form:

$$
-\beta \phi_{n}+\left(1+\delta_{n, n_{0}} \nu\left|\phi_{n}\right|^{2}\right)\left(\phi_{n+1}+\phi_{n-1}\right)=0
$$

where, $\beta \equiv \omega / V$.

We will focus on two special cases, (i) Impurity in the "bulk" and (ii) "surface" impurity.

Impurity in the "bulk": In this case, $-\infty<n<\infty$ and without loss of generality, we choose $n_{0}=0$. We pose a solution in the form $\phi_{n}=A \xi^{|n|}$, where $0<|\xi|<1$. After inserting this ansatz into Eq.(8) , one obtains $\beta=2 \xi\left(1+\nu A^{2}\right)$ and $\beta=\xi+(1 / \xi)$. After solving for $\xi$, one obtains

$$
\xi^{2}=\frac{1}{1+2 \nu A^{2}}
$$

On the other hand, from the normalization condition, Eq.(7), one obtains the relation

$$
1=\frac{1}{\nu} \log \left(1+\nu A^{2}\right)+\frac{2 A^{2} \xi^{2}}{1-\xi^{2}}
$$



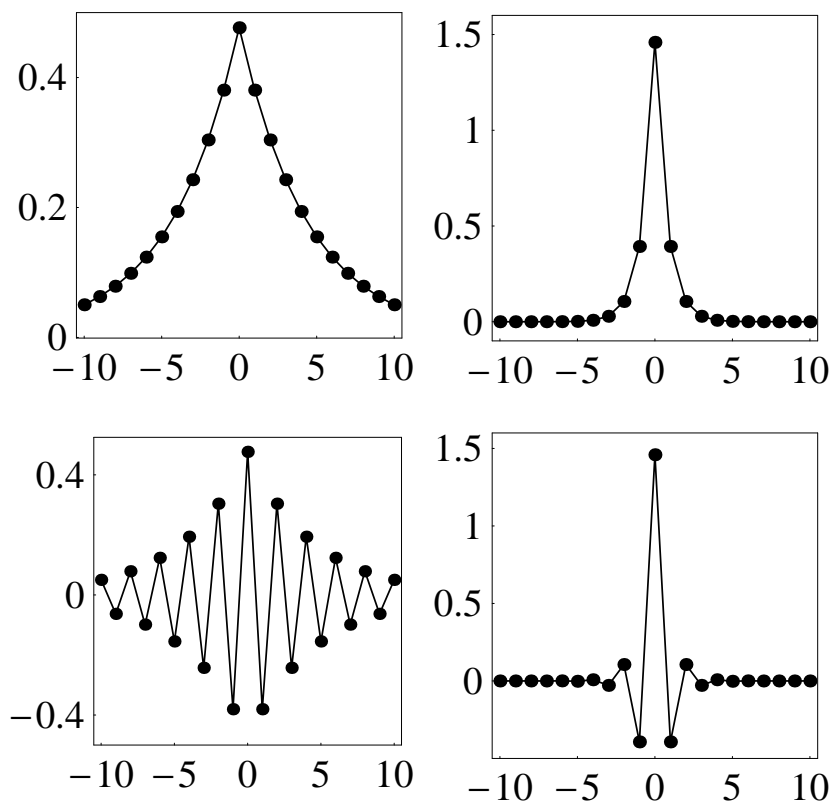

FIG. 1: Impurity in bulk: localized modes for $\nu=1.25$ (left column) and $\nu=1.5$ (right column). The top (bottom) row shows the unstaggered (staggered) versions of the mode.

After combining these last two equations, one obtains $\xi= \pm[2 \exp (\nu-1)-1]^{-1 / 2}$, and $A=((\exp (\nu-1)-1) / \nu)^{1 / 2}$, which implies

$$
\phi_{n}=( \pm 1)^{n}\left(\frac{\exp (\nu-1)-1}{\nu}\right)^{1 / 2}(2 \exp (\nu-1)-1)^{-|n| / 2} .
$$

The dimensionless bound state energy is

$$
\beta= \pm\left([2 \exp (\nu-1)-1]^{-1 / 2}+[2 \exp (\nu-1)-1]^{1 / 2}\right)
$$

As can be seen from Eq.(11), a localized bound state is possible provided $\nu>\nu_{c}=1$, and for a given $\nu$, there is an unstaggered (staggered) version of the bound state for $\beta>$ $2(<-2)$. Fig.1 shows a couple of profiles $\phi_{n}$ and their staggered versions, for two different dimensionless nonlinearity parameters $\nu$. In Fig.2 we show $\xi$ and the bound state energy as a function of nonlinearity. Standard linear stability analysis reveals that this stationary localized state is stable.

An interesting feature arises when we consider the dynamical excitation of a localized state. In this case, one considers Eq.(6) for a highly localized initial condition, chosen as $\phi_{n}(0)=\delta_{n, 0} \sqrt{(\exp (\nu)-1) / \nu}$. This choice corresponds to the one that saturates the normalization condition, Eq.(17). Examination of the ensuing dynamics reveals that at low 

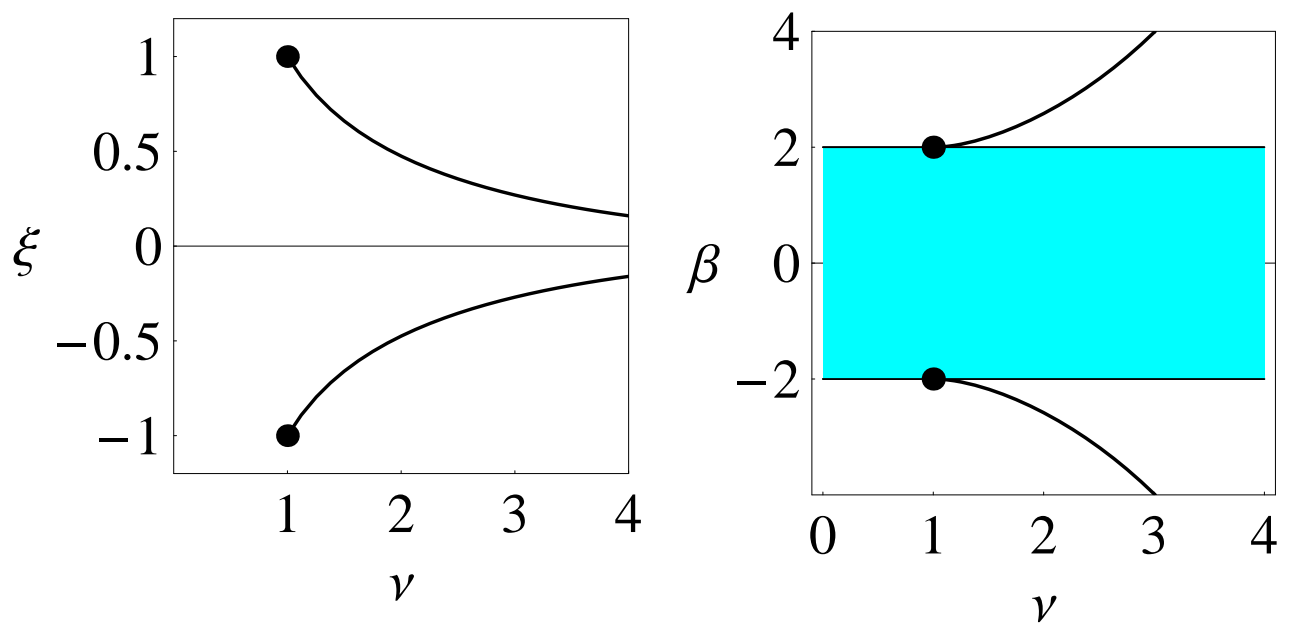

FIG. 2: Impurity in bulk. Left: $\xi$ as a function of $\nu$ for localized mode. Right: Bound state energy of localized mode as a function of nonlinearity parameter. The shaded area marks the position of the linear band, while the upper (lower) curve corresponds to the unstaggered (staggered) mode. The black dot marks the position of $\nu_{c}=1$.

nonlinearity values, the excitation tends to diffract across the array, while for higher nonlinearities, it tends to selftrap at the impurity site, with a high-amplitude oscillation, as Fig.3 clearly shows. The magnitude and frequency of these oscillations increase as the nonlinearity parameter $\nu$ is increased. We have checked numerically the persistence of this breathing phenomenon for long times, and believe that it can be understood from the special form of the normalization condition, Eq.(7): A small change in the sum of the square amplitudes at sites other than the impurity site will bring about a large change of the amplitude at the impurity site, due to the logarithmic dependency of the latter. To be more precise, let us assume that shortly after launching the initial excitation, a certain amount of radiation is emitted causing $\sum^{\prime}\left|\phi_{n}\right|^{2} \rightarrow \sum^{\prime}\left|\phi_{n}\right|^{2}-\Delta$; then it can be easily proven that $\left|\phi_{0}\right|^{2} \rightarrow\left|\phi_{0}\right|^{2}+(1 / \nu)(\exp (\nu \Delta)-1)$. Thus, it is the particular form of the AL nonlinearity that amplifies the breathing oscillations exponentially at the impurity site. We have also computed the long-time average probability at the initial site, as a function of nonlinearity strength. For our relatively short chain (100 sites), there is no sharp selftrapping threshold, although there is an inflexion point around $\nu=7$, as Fig. 3 shows.

Surface impurity: We now consider the case when the impurity is at the very beginning of a semi-infinite lattice. We relabel the previous chain, so that the first site is now at $n_{0}=0$. 

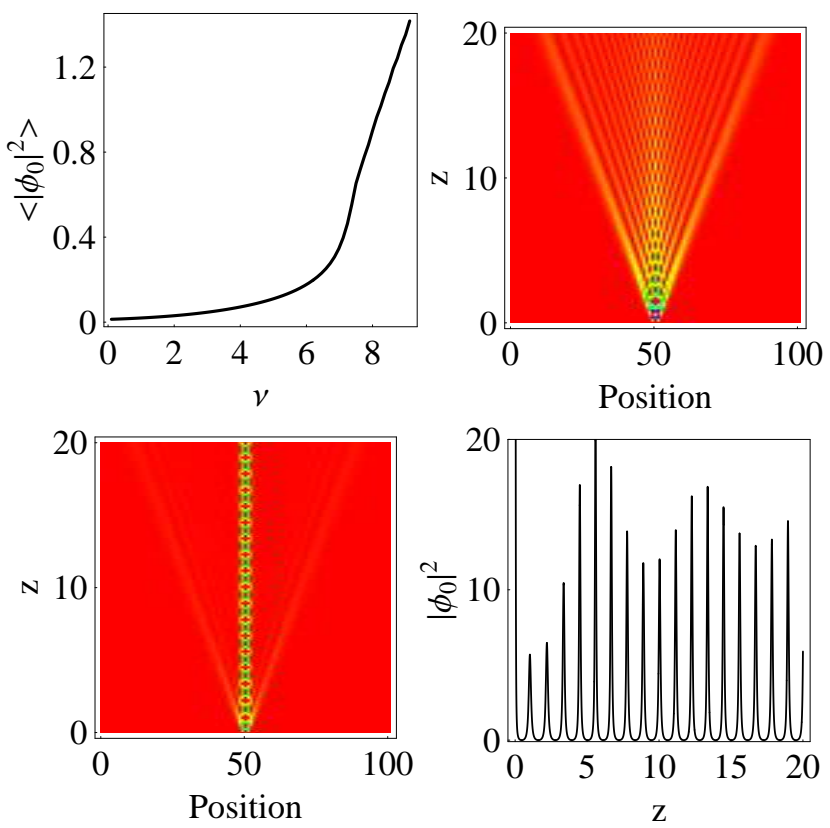

FIG. 3: Impurity in bulk. Top left: Long-time average probability at impurity site. Top right: Evolution of initial localized excitation across the lattice for $\nu=2$. Bottom left: Evolution for $\nu=8$. Bottom right: Evolution of amplitude at impurity site for $\nu=8$.

The dimensionless stationary-state equations read now

$$
\begin{gathered}
-\beta \phi_{0}+\left(1+\nu\left|\phi_{0}\right|^{2}\right) \phi_{1}=0 \\
-\beta \phi_{n}+\left(\phi_{n+1}+\phi_{n-1}\right)=0, \quad n=0,1,2, \ldots
\end{gathered}
$$

We proceed as before and pose a solution of the form $\phi_{n}=A \xi^{n}$, where $0<|\xi|<1$ and $n=0,1,2, \ldots$. After replacing this ansatz into Eq.(13) and (14), one obtains $\beta=\left(1+\nu A^{2}\right) \xi$ and $\beta=\xi+(1 / \xi)$, which implies

$$
\xi^{2}=\frac{1}{\nu A^{2}}
$$

On the other hand, from the normalization condition, Eq.(7), we have

$$
1=\frac{1}{\nu} \log \left(1+\nu A^{2}\right)+\frac{A^{2} \xi^{2}}{1-\xi^{2}}
$$

From Eqs. (15) and (16), we obtain a transcendental equation for $\xi$ :

$$
\nu=\log \left(1+\frac{1}{\xi^{2}}\right)+\frac{1}{1-\xi^{2}}
$$


Simple analysis shows that there is a critical nonlinearity value $\nu_{c}=(3 / 2)+\log (4) \approx 2.9$, such that, for $\nu<\nu_{c}$ there is no bound state, at $\nu=\nu_{c}$ there is exactly one bound state, while for $\nu>\nu_{c}$ there are two bound states. One of these states, becomes more narrow and its energy detaches from the linear band as nonlinearity is increased, while the second one becomes wider and its energy approaches the linear band upon increase in nonlinearity (see Fig.5 below). Straightforward linear stability analysis reveals that the former state is stable, while the latter is unstable.

The bound state mode is given by

$$
\phi_{n}=\frac{1}{\sqrt{\nu}} \xi(\nu)^{n-1} \quad n=0,1, \ldots
$$

where $\xi$ has to be found numerically from Eq.(17), for a given $\nu>\nu_{c}$. It is possible, however, to derive a very simple, yet accurate, approximation for $\xi=\xi(\nu)$, as follows: We start from Eq.(17) re-written as

$$
\exp (\nu)=\left(\frac{1+\xi^{2}}{\xi^{2}}\right) \exp \left(1 /\left(1-\xi^{2}\right)\right)
$$

Now, since $0<|\xi|<1$, it makes sense to expand around $\xi=0$. To fourth-order in $\xi$, Eq.(19]) becomes

$$
\xi^{2} e^{\nu-1} \approx 1+2 \xi^{2}+(5 / 2) \xi^{4}
$$

which implies,

$$
\xi(\nu) \approx \pm\left(\frac{1}{5}\left(\exp [\nu-1]-2-(\exp [2(\nu-1)]-4 \exp [\nu-1]-6)^{1 / 2}\right)\right)^{1 / 2}
$$

Numerical comparison with the exact value, reveals that the relative percentage error of approximation (21) is less than $3 \%$ for $\nu>3$.

Figure 4 shows some amplitude profiles in the vicinity of the lattice surface for a couple of different $\nu$ values. Figure 5 shows the numerical solution for $\xi$ and the localized state energy as a function of nonlinearity. As before, values of $\beta$ above (below) the band give rise to unstaggered (staggered) states.

Comparison between Figs. 2 and 5 reveals that, as far as stationary localized modes is concerned, the presence of a surface increases the minimum amount of nonlinearity needed to create a bound state. The boundary is acting as a repulsive surface, similar to what has been observed earlier in semi-infinite DNLS systems[4, 6]

Finally, we examine the dynamics of an excitation initially localized at the surface of the system $n=0$. The idea is to determine how the presence of a boundary affects the dynamical 
creation of a surface localized mode. As before, we take $\phi_{n}(0)=\sqrt{(\exp (\nu)-1) / \nu} \delta_{n, 0}$ and examine the average probability remaining at the initial site for long times, as well as the behavior of the amplitude at the impurity. Results are displayed in Fig.6, which is qualitatively similar to its bulk counterpart, Fig.3. As before, we observe diffraction behavior for small nonlinearity values and selftrapping at large $\nu$ values (at approximately $\nu \sim 14.6)$. In the last case, we also observe large-amplitude oscillations at the impurity site. The main difference with the bulk case, is that we need now substantially larger $\nu$ values to effect selftrapping.

In conclusion, we have examined the stationary-state and dynamical localized modes residing on a AL-like impurity, embedded well inside and at the surface of a one-dimensional discrete lattice. For both cases, the dynamical localized mode displays high-amplitude (exponential) oscillations at the impurity site, due to the particularly asymmetric form of the coupling between the impurity and its neighbors. The presence of a surface, on the other hand, increases the amount of minimum nonlinearity needed to create a localized mode, in agreement with previous studies on one-dimensional DNLS systems.
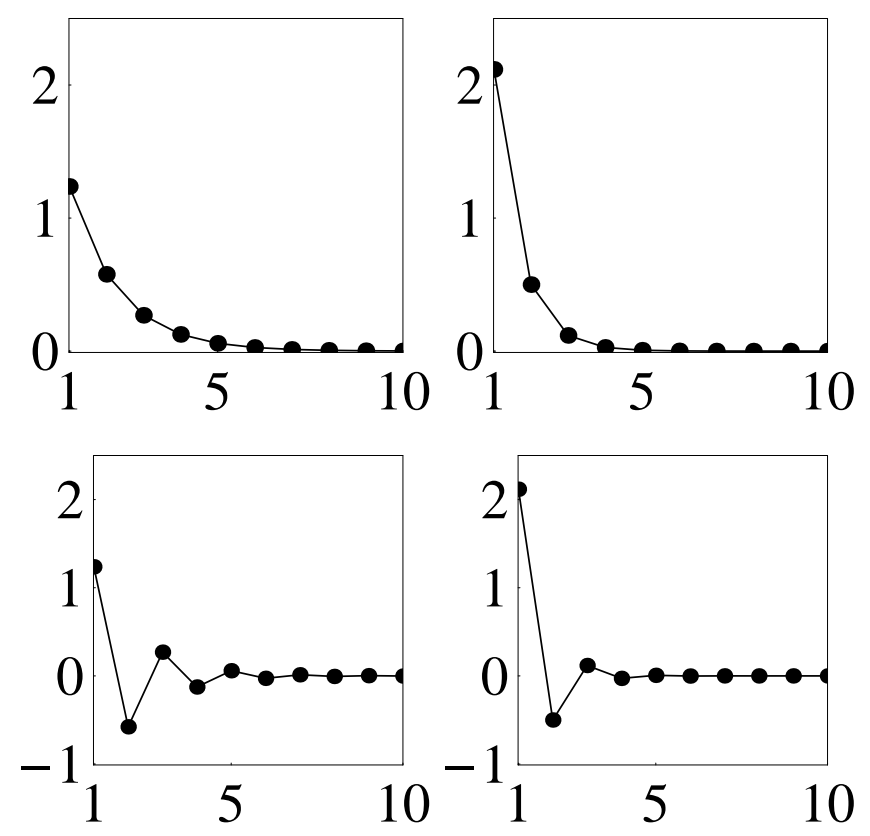

FIG. 4: Surface impurity: localized modes for $\nu=3$ (left column) and $\nu=4$ (right column). The top (bottom) row shows the unstaggered (staggered) versions of the mode. 

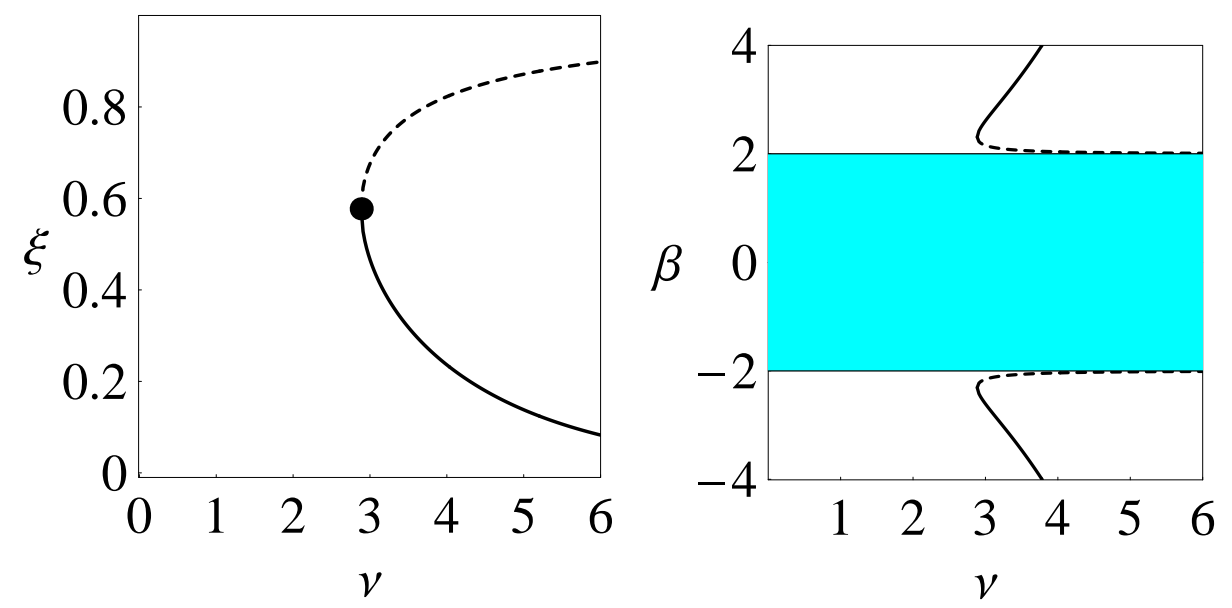

FIG. 5: Surface impurity. Left: Numerical solution for $\xi$ in terms of $\nu$. Right: Bound state energies of localized modes as a function of nonlinearity parameter. Solid(dashed) curve denotes stable(unstable) solution.
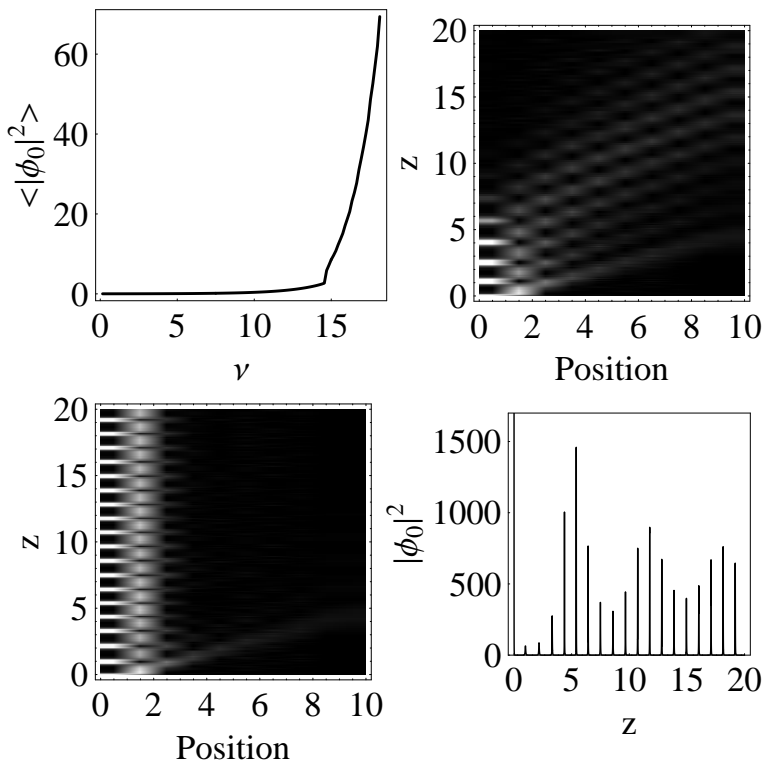

FIG. 6: Surface impurity. Top left: Long-time average probability at impurity site. Top right: Evolution of initial localized excitation across the lattice for $\nu=14$. Bottom left: Evolution for $\nu=15$. Bottom right: Evolution of amplitude at impurity site for $\nu=15$. 


\section{ACKNOWLEDGMENTS}

This work has been supported by Fondecyt grant 1080374 in Chile. The author is grateful to M. J. Ablowitz for useful discussions.

[1] See, e.g., D.N. Christodoulides, F. Lederer, and Y. Silberberg, Nature 424, 817 (2003) and references therein.

[2] M. J. Ablowitz and J. F. Ladik, J. Math. Phys. 16, 598, (1975).

[3] J. Gernier, Phys. Rev. E 63, 026608 (2001).

[4] M. I. Molina, Phys. Rev. B 71, 035404 (2005).

[5] K. G. Makris, S. Suntsov, D. N. Christodoulides and G. I. Stegeman, Opt. Lett. bf 30, 2466 (2005).

[6] M. I. Molina, R. A. Vicencio and Y. S. Kivshar, Optt. Lett. 31, 1693 (2006).

[7] M. I. Molina, Phys. Rev. B 74, 045412 (2006).

[8] H. Susanto, P. G. Kevrekidis, B. A. Malomed, R. Carretero-González and D. J. Frantzeskakis, Phys. Rev. E. 75, 056605 (2007). 


\section{List of Figure Captions}

Figure 1: Impurity in bulk: localized modes for $\nu=1.25$ (left column) and $\nu=1.5$ (right column). The top (bottom) row shows the unstaggered (staggered) versions of the mode.

Figure 2: (Color online) Impurity in bulk. Left: $\xi$ as a function of $\nu$ for localized mode. Right: Bound state energy of localized mode as a function of nonlinearity parameter. The shaded area marks the position of the linear band, while the upper (lower) curve corresponds to the unstaggered (staggered) mode. The black dot marks the position of $\nu_{c}=1$.

Figure 3: (Color online) Impurity in bulk. Top left: Long-time average probability at impurity site. Top right: Evolution of initial localized excitation across the lattice for $\nu=2$. Bottom left: Evolution for $\nu=8$. Bottom right: Evolution of amplitude at impurity site for $\nu=8$.

Figure 4: Surface impurity: localized modes for $\nu=3$ (left column) and $\nu=4$ (right column). The top (bottom) row shows the unstaggered (staggered) versions of the mode.

Figure 5: (Color online) Surface impurity. Left: Numerical solution for $\xi$ in terms of $\nu$. Right: Bound state energies of localized modes as a function of nonlinearity parameter. Solid(dashed) curve denotes stable(unstable) solution.

Figure 6: Surface impurity. Top left: Long-time average probability at impurity site. Top right: Evolution of initial localized excitation across the lattice for $\nu=14$. Bottom left: Evolution for $\nu=15$. Bottom right: Evolution of amplitude at impurity site for $\nu=15$. 